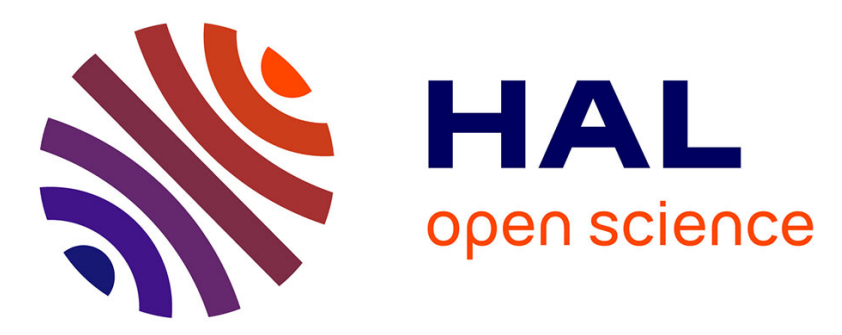

\title{
An improved method to design QPSK Balanced Space-Time Trellis Codes
}

Pierre Viland, Gheorghe I. Zaharia, Jean-François Hélard

\section{To cite this version:}

Pierre Viland, Gheorghe I. Zaharia, Jean-François Hélard. An improved method to design QPSK Balanced Space-Time Trellis Codes. Wireless Conference, 2008, Jun 2008, Prague, Czech Republic. pp. 1-6, 10.1109/EW.2008.4623852 . hal-00348787

\section{HAL Id: hal-00348787 https://hal.science/hal-00348787}

Submitted on 22 Dec 2008

HAL is a multi-disciplinary open access archive for the deposit and dissemination of scientific research documents, whether they are published or not. The documents may come from teaching and research institutions in France or abroad, or from public or private research centers.
L'archive ouverte pluridisciplinaire HAL, est destinée au dépôt et à la diffusion de documents scientifiques de niveau recherche, publiés ou non, émanant des établissements d'enseignement et de recherche français ou étrangers, des laboratoires publics ou privés. 


\title{
An improved method to design QPSK Balanced Space-Time Trellis Codes
}

\author{
Pierre Viland Gheorghe Zaharia Jean-François Hélard \\ Institute for Electronics and Telecommunications of Rennes (IETR) - UMR CNRS 6164 \\ INSA - 20 avenue des Buttes de Coësmes, \\ 35043 Rennes, France \\ Email: pierre.viland@ens.insa-rennes.fr
}

\begin{abstract}
Recently, a new class of Space-Time Trellis Codes was proposed as having the best performance. These codes are 'balanced' because they use the points of the constellation with the same probability. In this correspondence, we propose a new and simpler method than exisiting method to design these class for QPSK modulation and several transmit antennas. New and better balanced codes for 3 and 4 transmit antennas are also proposed.
\end{abstract}

Keywords: space-time trellis codes, balanced codes, MIMO systems, design method.

\section{INTRODUCTION}

Space Time Trellis Coded Modulation (STTCM) was introduced in 1998 by Tarokh et al. [1] by combining channel coding with the Multiple Input Multiple Output (MIMO) concept to improve the data rate and the reliability of wireless communications. Many performance criteria have been established to maximize both diversity and coding gain of STTC. The rank and determinant criteria for slow fading channels with the Euclidian distance and the product distance criteria for fast fading channels have been proposed in [1]. In [2] Chen introduced the trace criterion which governs the coding for systems with a great product of the numbers of transmit $(\mathrm{Tx})$ and receive $(\mathrm{Rx})$ antennas.

Based on above criteria, many different STTC for 2 Tx antennas have been found by a systematic code search [3-6]. The performance study of these codes was carried out over slow and fast Rayleigh fading channels to identify the most efficient ones [7]. It has been shown that over slow fading channels, the codes constructed with the trace criterion give similar or even better results that the codes constructed with the rank and the determinant criteria. Over fast fading channels, "trace criterion codes" as Chen's codes outperform the other tested codes. In the same way, some codes for $3 \mathrm{Tx}$ antennas have been published in [5], [8]. One can remark that all the codes which achieve the best performance have the same property: they use the points of the constellation with the same probability if the data are generated by a binary memoryless source with equally probable symbols. Therefore, these codes where called "Balanced-STTC" (B-STTC) [9]. A first construction method of B-STTC has been proposed in [911]. Thus, the search of good STTCs can be reduced to this class. In this paper, a new and simple method which can be easly generalised for $2^{\mathrm{n}}$-PSK and $n_{T}$ transmit antenna is given. Besides, new Balanced STTC are presented with better performance than the previously published codes.

The rest of the paper is organised as follows: section 2 presents briefly the Space-Time Trellis Coding. In section 3, the performance criteria of STTC are reminded. In section 4, the properties of balanced codes are introduced. Examples of published balanced codes are given It is also shown that most of them are balanced. In section 5, a method to design BSTTC is presented for QPSK modulation and $n_{T}$ transmit antennas. Finally, the paper compares the performance of published codes and new optimal balanced codes.

\section{SPACE TIME TRELLIS CODING}

We consider the general case of $2^{n}$-PSK space-time trellis encoder. Fig. 1 shows this encoder for $n=2$.

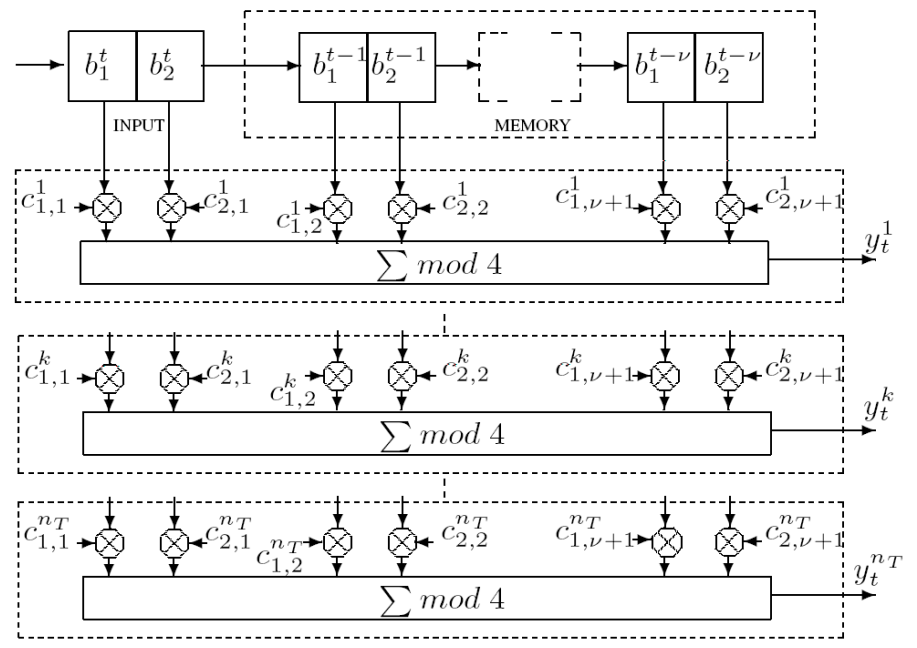

Figure 1. ST trellis encoder with 4-PSK and $\mathrm{n}_{\mathrm{T}}$ antennas.

This encoder has one input block of $\mathrm{n}$ bits and $v$ memory blocks of $\mathrm{n}$ bits. At each time $t \in \mathbb{Z}$, the bits of a block are replaced by the $\mathrm{n}$ bits of the previous block. The $i^{\text {th }}$ bit $b_{i}^{t-j+1}, i=1 \ldots n$, of the $j^{t h}$ block, $j=1 \ldots(v+1)$, is associated to $\mathrm{n}_{\mathrm{T}}$ multiplier coefficients $c_{i, j}^{k} \in \mathbb{Z}_{2^{n}}, k=1 \ldots n_{T}$, 
where $n_{T}$ is the number of transmit antennas. A ST trellis encoder is thus usually defined by its generator matrix $C$ of $n_{T} \times n(v+1)$ coefficients:

$$
C=\left[\begin{array}{ccccccc}
c_{1,1}^{1} & \ldots & c_{n, 1}^{1} & \ldots & c_{1, v+1}^{1} & \ldots & c_{n, v+1}^{1} \\
\vdots & & \ldots & & \ldots & & \vdots \\
c_{1,1}^{k} & \ldots & c_{n, 1}^{k} & \ldots & c_{1, v+1}^{k} & \ldots & c_{n, v+1}^{k} \\
\vdots & & \ldots & & \ldots & & \vdots \\
c_{1,1}^{n_{T}} & \ldots & c_{n, 1}^{n_{T}} & \ldots & c_{1, v+1}^{n_{T}} & \ldots & c_{n, v+1}^{n_{T}}
\end{array}\right]
$$

The encoder outputs for the $k^{\text {th }}$ antenna are computed as

$$
y_{t}^{k}=\sum_{i=1}^{n} \sum_{j=1}^{v+1} b_{i}^{t-j+1} c_{i, j}^{k} \quad \bmod 2^{\mathrm{n}}
$$

and sent to the $k^{\text {th }}$ antenna. The modulated streams for all antennas are then transmitted simultaneously.

\section{DESIGN CRITERIA}

Design criteria have been proposed in [1-2] to exploit the spatial diversity and to offer optimal coding gain. Cases of slow and fast Rayleigh fading channels are mainly studied. The transmitted $n_{T}$ dimension symbols $s_{t}=\left[s_{t}^{1} s_{t}^{2} \ldots s_{t+L_{f}+1}^{n_{T}}\right]^{T}$, where $[\cdot]^{T}$ denotes the transpose operator, are assumed to be grouped in a frame of length $L_{f}$. For each case, criteria are derived from the minimization of the Pairwise Error Probability (PEP), i.e. the probability of transmitting the $n_{T} \times L_{f}$ dimension coded frame $s_{t}=\left[s_{t} s_{t} \ldots s_{t+L_{f}+1}\right]^{T}$ and deciding erroneously in favour of another $n_{T} \times L_{f}$ dimension coded frame $e_{t}=\left[e_{t}^{1} e_{t}^{2} \ldots e_{t+L_{f}+1}^{n_{T}}\right]^{T}$. The $n_{T} \times n_{T}$ product matrix $\mathbf{A}=\mathbf{B} . \mathbf{B}^{*}$ is introduced, where $\mathbf{B}^{*}$ denotes the hermitian of the $n_{T} \times L_{f}$ difference matrix $\mathbf{B}=\mathbf{E}-\mathbf{S}$.

$$
B=\left[\begin{array}{ccccc}
e_{t}^{1}-s_{t}^{1} & \ldots & e_{t+q}^{1}-s_{t+q}^{1} \ldots & e_{t+L_{f}-1}^{1}-s_{t+L_{f}-1}^{1} \\
\ldots & \ldots & \ldots & \ldots & \ldots \\
e_{t}^{n_{T}}-s_{t}^{n_{T}} \ldots & \ldots & e_{t+q}^{n_{T}}-s_{t+q}^{n_{T}} \ldots & \ldots & e_{t+L_{f}-1}^{n_{T}}-s_{t+L_{f}-1}^{n_{T}}
\end{array}\right]
$$

For slow Rayleigh fading, if $\operatorname{rank}(A) \cdot n_{R} \leq 3$, two criteria are proposed [1], [12]:

- A has to be a full rank matrix for any codeword $c$ and $e$.

- The coding gain is given by: $\eta=\sum_{d} N(d) d^{-n_{R}}$ where $N(d)$ is defined as the average number of error events with determinant $d$. The best code must has the minimum value of $\eta$.

In [2], Chen proposes a new criterion valid in the case of slow and fast Rayleigh fading channels if $\operatorname{rank}(A) n_{R}>3$. Under this assumption, the PEP is minimized if the sum of all the eigenvalues of the product matrix is maximized. For a square matrix, the sum of all the eigenvalues is equal to the trace of the matrix A.

$$
\operatorname{tr}(A)=\sum_{k=1}^{n_{T}} \lambda_{k}=\sum_{k=1}^{n_{T}}\left(\sum_{q=0}^{L_{f}-1}\left|e_{t+q}^{k}-s_{t+q}^{k}\right|^{2}\right)
$$

For each pair of coded frames, the matrix $\boldsymbol{A}$ and then $\operatorname{tr}(\boldsymbol{A})$ can be computed.The minimum trace is the minimum of all these values $\operatorname{tr}(\mathbf{A})$. When $\operatorname{rank}(\mathbf{A}) n_{R}>3$, the minimization of the PEP amounts to use a code which has the maximum value of the minimum trace. In [12], it is also stated that to minimize the FER, the number of error events with minimum trace has to be minimized.

\section{BALANCED CODES}

\subsection{What is a 'balanced code'?}

This concept of 'balanced codes' has been proposed in [9-11]. It is based on the observation that the best STTC proposed in the literature present the same property: the generated symbols of the MIMO constellation are equally probable.

If the binary input data is generated by a memoryless binary source $S=\{0,1\}$ with equally probable symbols, then, in the case of $2^{\text {n}}$-PSK modulation, from a given state $\boldsymbol{X}=\left[x_{1} x_{2} \ldots x_{L}\right]^{T} \in \mathbb{Z}_{2}^{L}$ of the $L=n(v+1)$ length shift register realized by $(\mathrm{v}+1)$ blocks of $\mathrm{n}$ bits, the MIMO symboles $\boldsymbol{Y}=\left[y_{1} y_{2} \ldots y_{n_{T}}\right]^{T} \in \mathbb{Z}_{2^{n}}^{n_{T}}$ generated by the STTC encoder shown in fig. 1 is

$$
\boldsymbol{Y}=\boldsymbol{G} \boldsymbol{X}
$$

where $G$ is the generator matrix (1). This is a deterministic relation. Therefore, the STTC is defined by a map :

$$
\Phi: \mathbb{Z}_{2}^{L} \rightarrow \mathbb{Z}_{2^{n}}^{n_{T}}
$$

which associates to the state $X$ a unique codeword $Y$. Note that $\Phi\left(\mathbb{Z}_{2}^{L}\right) \subseteq \mathbb{Z}_{2^{n}}^{n_{T}}$ represents the set of generated codewords $Y$.

By definition, a STTC is balanced, if and only if each generated codeword $Y \in \Phi\left(\mathbb{Z}_{2}^{L}\right)$ has the same number of occurrences:

$$
n(Y)=n_{0} \in \mathbb{N}^{*}, \forall Y \in \mathbb{Z}_{2^{n}}^{n_{T}}
$$

If $\Phi\left(\mathbb{Z}_{2}^{L}\right)=\mathbb{Z}_{2^{n}}^{n_{T}}$ the code is fully balanced i.e. $\left\{\sum_{j=1}^{j=L}\left(x_{j} G_{j}\right) \bmod 2^{n} / x_{j} \in\{0,1\}\right\}=\mathbb{Z}_{2^{n}}^{n_{T}}$ where $G_{j}$ is the $\mathrm{j}^{\text {th }}$ column of the matrix $\boldsymbol{G}$.

$$
\text { If } \operatorname{card}\left(\Phi\left(\mathbb{Z}_{2}^{L}\right)\right)=\frac{\operatorname{card}\left(\Phi\left(\mathbb{Z}_{2^{n}}^{n_{T}}\right)\right)}{2} \text { and each } Y \in \Phi\left(\mathbb{Z}_{2}^{L}\right)
$$

has the same number of occurrences, the code is half balanced. 
The number of different codewords generated by relation (5) is given by $\operatorname{card}\left(\Phi\left(\mathbb{Z}_{2}^{L}\right)\right)$ which is a power of 2 .

Due to the random memoryless source $\mathbf{S}=\{0,1\}$ with $\mathrm{p}(0)=\mathrm{p}(1)=1 / 2$, from a given state $\boldsymbol{X}$, the shift-register the encode can has only $2^{n}$ equally probable next states. The matrix $\mathbf{T}$ of the transition probabilities between these states corresponds to a Markov chain. Due to the symmetry of the matrix $\mathbf{T}$, the steady-state probabilities of the states $\boldsymbol{X}$ are all equal. For a balanced code, by using (5), the generated codewords $\boldsymbol{Y}$ are also equally probable. In other words, the generated symbols of the constellation are equally probable.

\subsection{Published codes}

Most of the good published and best STTCs belong to the class of balanced codes. In this section, a no exhaustive list of known codes is presented for some configurations of STTCs. In table I, several known QPSK codes, 2 transmit antennas with 4-state and 16 states are presented. It is easy to verify that all these codes are fully balanced. For each 4 -state code given in Table 1, the 4 columns $G_{j}$ of the generator matrix $\boldsymbol{G}$ create the group $\mathbb{Z}_{4}^{2}$ i.e. $\left\{\sum_{j=1}^{j=4}\left(x_{j} G_{j}\right) \bmod 4 / x_{j} \in\{0,1\}\right\}=\mathbb{Z}_{4}^{2}$. Therefore, for each $X \in \mathbb{Z}_{2}^{4}$, a different $Y \in \mathbb{Z}_{4}^{2}$ is generated, so $n(Y)=1 \quad \forall Y \in \mathbb{Z}_{4}^{2}$. The 16-state code are also fully balanced and each point of constellation, is used 4 times, so $n(Y)=4 \forall Y \in \mathbb{Z}_{4}^{2}$.

TABLE I. SOME KNOWN QPSK B-STTC $\left(\mathrm{n}_{\mathrm{T}}=2\right)$

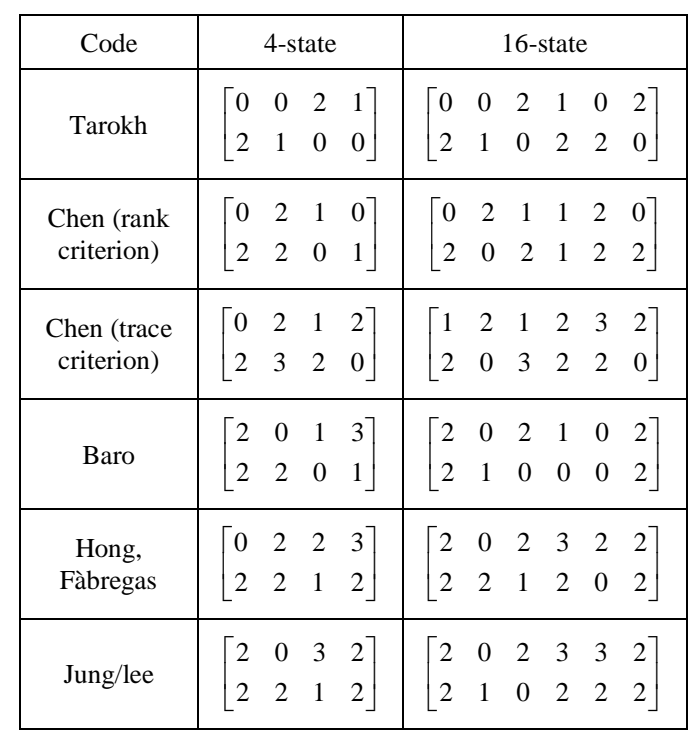

Table II shows some QPSK STTCs for 3 and 4 Tx antennas proposed by Chen in [8] and Bernierin [13]. In this case, fully balanced codes generate respectively the elements of $\mathbb{Z}_{4}^{3}$ and $\mathbb{Z}_{4}^{4}$ with the same number of occurances: $\mathrm{n}(\mathrm{Y})=1, \forall Y \in \mathbb{Z}_{4}^{3}$ and $\forall Y \in \mathbb{Z}_{4}^{4}$. The codes noted by "NB" are no-balanced, those noted by "B" are balanced and those noted by "'FB" are fully balanced.

TABLE II. SOME KNOWN QPSK STTC $\left(\mathrm{n}_{\mathrm{T}}=3\right.$ and $\left.\mathrm{n}_{\mathrm{T}}=4\right)$

\begin{tabular}{|c|c|c|c|c|c|c|c|c|c|c|c|c|c|c|}
\hline $\mathrm{n}_{\mathrm{T}}$ & States & Code & \multicolumn{11}{|c|}{ G } & \\
\hline \multirow[t]{4}{*}{3} & \multirow[t]{2}{*}{16} & $\begin{array}{c}\text { Chen (trace } \\
\text { criterion) }\end{array}$ & & & & 2 & 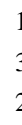 & & & $\begin{array}{l}3 \\
2\end{array}$ & & & & B \\
\hline & & $\begin{array}{l}\text { Chen (rank } \\
\text { criterion) }\end{array}$ & & & 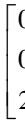 & 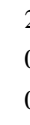 & 1 & & $\frac{1}{2}$ & $\begin{array}{l}2 \\
3 \\
1\end{array}$ & $\begin{array}{l}2 \\
3 \\
3\end{array}$ & & & NB \\
\hline & 64 & $\begin{array}{l}\text { Chen (trace } \\
\text { criterion) }\end{array}$ & & 2 & 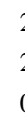 & 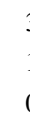 & 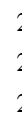 & & 3 & $\begin{array}{l}0 \\
0 \\
3\end{array}$ & $\begin{array}{l}3 \\
2 \\
1\end{array}$ & $\begin{array}{l}2 \\
0 \\
1\end{array}$ & & FB \\
\hline & 256 & Bernier & 2 & 2 & 3 & 2 & 0 & & 2 & $\begin{array}{l}2 \\
2 \\
2\end{array}$ & $\begin{array}{l}2 \\
1 \\
1\end{array}$ & $\begin{array}{l}0 \\
2 \\
2\end{array}$ & $\left.\begin{array}{l}2 \\
2 \\
0\end{array}\right]$ & FB \\
\hline 4 & 16 & $\begin{array}{l}\text { Chen (trace } \\
\text { criterion) }\end{array}$ & & & & 2 & 2 & & $\begin{array}{l}2 \\
2 \\
0\end{array}$ & $\begin{array}{l}2 \\
1 \\
3\end{array}$ & $\begin{array}{l}2 \\
0 \\
2 \\
2\end{array}$ & & & B \\
\hline & 64 & $\begin{array}{l}\text { Chen (trace } \\
\text { criterion) }\end{array}$ & & $\begin{array}{l}2 \\
2 \\
1\end{array}$ & 2 & 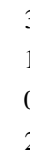 & 2 & & $\begin{array}{l}3 \\
3 \\
2\end{array}$ & $\begin{array}{l}0 \\
0 \\
3 \\
1\end{array}$ & $\begin{array}{l}3 \\
2 \\
1 \\
3\end{array}$ & $\begin{array}{l}2 \\
0 \\
1 \\
2\end{array}$ & & NB \\
\hline & 128 & Bernier & $\begin{array}{l}2 \\
3 \\
3\end{array}$ & 2 & 2 & 3 & 3 & & 2 & $\begin{array}{l}0 \\
1 \\
2\end{array}$ & $\begin{array}{l}2 \\
0\end{array}$ & $\begin{array}{l}1 \\
2 \\
1 \\
1\end{array}$ & $\left.\begin{array}{l}0 \\
0 \\
0 \\
0\end{array}\right]$ & FB \\
\hline
\end{tabular}

There exists some no-balanced published codes which have good performance. However, it is possible to find better codes in the class of balanced codes. In fact, in a next section, for each published no-balanced code, an example of a balanced code which better performance is given.

\section{A GENERAL METHOD TO DESIGN QPSK BALANCED STTC WITH $\mathrm{n}_{\mathrm{T}}$ ANTENNAS}

\subsection{General properties of balanced codes}

Property 1: If a MIMO code wih a L-length shift register is fully balanced then $L \geq L_{\min }=n . n_{T}$. One can observe that $L_{\min }=\operatorname{dim}\left(\mathbb{Z}_{2^{n}}^{n_{T}}\right)$.

Property 2: Let us consider a fully balanced MIMO code with a $L$-length shift-register. Then, for any additional column matrix $G_{L+1} \in \mathbb{Z}_{2^{n}}^{n_{T}}$, the resulting MIMO code with a $(\mathrm{L}+1)$ length shift-register is also balanced.

Property 3: A code matrix $\boldsymbol{G}$ with $L_{m i n}$-length shift register is fully balanced if and only if the set of generated MIMO symbols $H_{L_{\min }}=\Phi\left(\mathbb{Z}_{2}^{L_{\min }}\right)$ is the group $\mathbb{Z}_{2^{n}}^{n_{T}}$ with i.e. : 
$\left\{\sum_{j=1}^{j=L_{\min }}\left(x_{j} G_{j}\right) \bmod 2^{n} / x_{j} \in\{0,1\}\right\}=\mathbb{Z}_{2^{n}}^{n_{T}}$. In this case, the set of columns $\left\{G_{l}, \ldots, G_{L_{\min }}\right\}$ is a base of $\mathbb{Z}_{2^{n}}^{n_{T}}$.

Property 4: $C_{[0]}=2 \mathbb{Z}_{2}^{n_{T}}$ is a subgroup of $\mathbb{Z}_{4}^{n_{T}}$ such as $v=-v, \forall v \in C_{[0]}$. Each element $v \in C_{[0]}$ can be written as $v=2 p$, with $p \in \mathbb{Z}_{2}^{n_{T}}$. The coset $C_{[p]}=p+C_{[0]}$ is called "relative to $v$ ".

\subsection{Design of QPSK fully balanced codes}

Due to Property 2, it is enough to design fully balanced codes with $L=L_{\text {min }}$. This new method to design fully balanced codes must respect the rules:

1. Choose a first no null column $G_{1}=2 p_{1} \in C_{[0]}$. Due to Property 4, $G_{1}=-G_{1}$, so $H_{1}=\left\{0, G_{1}\right\}$ is a subgroup of $\mathbb{Z}_{4}^{n_{T}}$.

2. Each new chosen column of $\boldsymbol{G}$ must contribute to create a new subgroup $H_{i+1}$ of $\mathbb{Z}_{4}^{n_{T}}$ with $\operatorname{card}\left(H_{i+1}\right)=2 \operatorname{card}\left(H_{i}\right)$.

Thus, in order to obtain a fully balanced STTC, the columns of $\mathrm{G}$ can be selected as follows:

- if $i<L_{\min }$ columns of $\boldsymbol{G}$ have been already chosen in $\mathbb{Z}_{4}^{n_{T}}$ and $\left\{\sum_{j=1}^{j=i} x_{j} G_{j} \bmod 4 / x_{j} \in\{0,1\}\right\}=H_{i}$ is a subgroup of $\mathbb{Z}_{4}^{n_{T}}$, then the column $G_{i+1}$ of $\boldsymbol{G}$ must belong to $\mathbb{Z}_{4}^{n_{T}} \backslash H_{i}$ and must be selected in cosets relative to vectors which belong to $C_{[0]} \cap H_{i}$. This method ensures that the created set $H_{i+1}=H_{i} \cup\left(H_{i}+G_{i+1}\right)$ is a subgroup of $\mathbb{Z}_{2^{n}}^{n_{T}}$. The algorithm ends when $i=L_{\text {min }}$.

Remark : It is easy to note that to create a fully balanced code with $L_{\text {min }}$-length shift register, the opposite elements of chosen and generated elements must not be selected because the sum between one element and its opposite is the identity element. Thus, in the method in [11], after the choice of one vector, the opposite of this element must be discarded (i.e. it must not be further selected). The new method has the advantage that for each chosen element, its opposite is also generated and so, no vector must be discarded. Thus, the construction of B-STTC is simplier than the method in [11]

In order to obtain balanced codes (not necessary fully balanced) the algorithm can be stopped at $\mathrm{i}_{0} \leq L_{\min }-2$. The obtained code is balanced but not fully balanced. One can add a new column $G_{i_{0}+1} \in \mathbb{Z}_{4}^{n_{T}} \backslash H_{i_{0}}$. Once again, the obtained code is balanced but not fully balanced. If necessary, several elements of $H_{i_{0}}$ can also be added as columns of the matrix $G$. The resulting code is also balanced but not fully balanced.

5.3. Example : design of fully balanced QPSK STTC with 2 transmit antennas

The partition of $\mathbb{Z}_{4}^{2}$ in 4 cosets is given in table III. TABLE III : PARTITION OF $\mathbb{Z}_{4}^{2}$ IN COSETS

\begin{tabular}{|c|c|c|c|c|}
\hline$C_{\left[\begin{array}{l}0 \\
0\end{array}\right]}$ & $\begin{array}{l}0 \\
0\end{array}$ & $\begin{array}{l}0 \\
2\end{array}$ & $\begin{array}{l}2 \\
0\end{array}$ & $\begin{array}{l}2 \\
2\end{array}$ \\
\hline$C_{[0}\left[\begin{array}{l}0 \\
1\end{array}\right]$ & $\begin{array}{l}0 \\
1\end{array}$ & $\begin{array}{l}0 \\
3\end{array}$ & $\begin{array}{l}2 \\
1\end{array}$ & $\begin{array}{l}2 \\
3\end{array}$ \\
\hline$C_{\left[\begin{array}{l}1 \\
0\end{array}\right]}$ & $\begin{array}{l}1 \\
0\end{array}$ & $\begin{array}{l}1 \\
2\end{array}$ & $\begin{array}{l}3 \\
0\end{array}$ & $\begin{array}{l}3 \\
2\end{array}$ \\
\hline$C_{\left[\begin{array}{l}1 \\
1\end{array}\right]}$ & $\begin{array}{l}1 \\
1\end{array}$ & $\begin{array}{l}1 \\
3\end{array}$ & $\begin{array}{l}3 \\
1\end{array}$ & $\begin{array}{l}3 \\
3\end{array}$ \\
\hline
\end{tabular}

The algorithm used to generate a fully balanced code with $L=L_{\min }$ is proceed as follows :

In agrement with rule 1 , the first no null vector $G_{1}=2 p_{1}$ must be selected in $C_{[0]}$. Thus, the subgroup $H_{1}=\left\{0, G_{1}\right\}$ of $\mathbb{Z}_{4}^{2}$ is generated. At least one element of $C_{[0]}$ must be chosen. Two types of B-STTC were introduced in [11] :

- Types I: codes with only 1 no null vector in $C_{[0]}$.

- Types II: codes with 2 no null vectors in $C_{[0]}$.

\section{For type I codes:}

The second element $G_{2}$ has to create a new subgroup $H_{2}=\left\{0, G_{1}, G_{2}, G_{1}+G_{2}\right\}$. For this type of code, because no other element of $C_{[0]}$ can be selected, therefore $G_{2} \in C_{\left[p_{1}\right]}$. The choice of $G_{3}$ must create a new subgroup $H_{3}=H_{2} \cup\left(H_{2}+G_{3}\right) \quad$ without adding a vector of $C_{[0]}$. Therefore $G_{3}$ must be also selected in $C_{\left[p_{1}\right]}$ among the two elements which do not belong to $H_{2}$. Due to the combinations between $G_{1}, G_{2}$ and $G_{3}, H_{3}=C_{[0]} \cup C_{\left[p_{1}\right]}$ is created. The last vector $G_{4}$ is selected among the two no generated cosets, i.e. $\mathbb{Z}_{4}^{2} \backslash H_{3}$. Thus, $\left\{G_{l}, \ldots, G_{4}\right\}$ is a base of $\mathbb{Z}_{4}^{2}$ and the matrix $\boldsymbol{G}$ of a fully QPSK B-STTC is obtained.

\section{For type II codes:}

The matrix $G$ of these codes have 2 no null columns $G_{1}, G_{2} \in C_{[0]}$, so $H_{2}=C_{[0]}$. It remains to select two different cosets between the 3 remaining cosets. In each chosen coset, one vector must be selected. Indeed, because $G_{3} \notin C_{[0]}$, $H_{3}=C_{[0]} \cup\left(C_{[0]}+G_{3}\right)$ is a new subgroup of $\mathbb{Z}_{4}^{2}$. Finally, because $G_{4} \notin H_{3}, \quad H_{4}=H_{3} \cup\left(H_{3}+G_{4}\right)=\mathbb{Z}_{4}^{2}$. 


\subsection{Example: design of fully balanced QPSK STTC with 3 transmit antennas}

For these codes, each generated element belongs to $\mathbb{Z}_{4}^{3}$. It is possible to make the partition of the additive group $\mathbb{Z}_{4}^{3}$ in a normal subgroup $C_{[0]}=2 \mathbb{Z}_{2}^{3}$ of $\mathbb{Z}_{4}^{3}$ and 7 cosets such as $C_{[p]}=p+C_{[0]}$ with $p \in \mathbb{Z}_{2}^{3} \backslash\left\{\left[\begin{array}{lll}0 & 0 & 0\end{array}\right]^{T}\right\}$.

In this case, there are three types of fully B-STTC [11]:

- Types I: codes with only 1 no null vector in $C_{[0]}$.

- $\quad$ Type II: codes with 2 no null vectors in $C_{[0]}$.

- Type III: codes with 3 no null vectors in $C_{[0]}$.

For type I codes:

Only the first column $G_{I}=2 p_{1}$ of $\boldsymbol{G}$ belongs to $C_{[0]}$. It generates $H_{1}=\left\{0, G_{1}\right\}$. According to the rule $2, G_{2}$ must be chosen in the coset $C_{\left[p_{1}\right]}$ relative to $G_{1}$. Thus, $\left\{G_{2}, G_{1}+G_{2}\right\}$ which is a quarter of $C_{\left[p_{1}\right]}$ is also generated. Because no new element of $C_{[0]}$ is generated, the third column must be selected in the same coset $C_{\left[p_{1}\right]}$. After the choice of this third column $G_{3} \in C_{\left[p_{1}\right]} \backslash H_{2}$, half of the coset $C_{\left[p_{1}\right]}$ and half of $C_{[0]}$ are generated. There are two solutions to choose the $4^{\text {th }}$ column:

1) $G_{4} \in C_{\left[p_{1}\right]} \backslash H_{3}$. In this case, the cosets $C_{\left[p_{1}\right]}$ and $C_{[0]}$ are completely generated. The column $G_{5}$ of $\boldsymbol{G}$ is selected in another coset. Then, two new cosets are generated and it remains four cosets to select the last column $G_{6}$.

2) $G_{4}$ is chosen in the cosets relative to the two new generated vectors $G_{2}+G_{3}=2 p_{2}$ and $G_{1}+G_{2}+G_{3}=2 p_{3}$ of $C_{[0]}$. After this choice, half of $C_{[0]}$ and half of three cosets $C_{\left[p_{1}\right]}, C_{\left[p_{2}\right]}$ and $C_{\left[p_{3}\right]}$ are generated. The $5^{\text {th }}$ vector $G_{5}$ is selected in the not generated half of the cosets $C_{\left[p_{2}\right]}$ and $C_{\left[p_{3}\right]}$. After the choice of $G_{5}, C_{[0]}$ and the three cosets are totally generated. The last column $G_{6}$ is chosen in one of the four other cosets.

\section{For type II codes:}

$G_{1}=2 p_{1}$ and $G_{2}=2 p_{2}$ generate $H_{2}=\left\{0, G_{1}, G_{2}, G_{1}+G_{2}\right\}$. The third column $G_{3}$ of $\boldsymbol{G}$ is selected in one of the three cosets $C_{\left[p_{1}\right]}, C_{\left[p_{2}\right]}$ and $C_{\left[p_{1} \oplus p_{2}\right]}$ (where $\oplus$ is the sum modulo 2 ) relative to the 3 no null generated elements of $C_{[0]}$. After this choice, half of the selected coset and half of $C_{[0]}$ are generated. The choice of the last three columns is similar to the case of type I codes.

\section{For type III codes:}

In this case, 3 no null columns $G_{1}, G_{2}$ and $G_{3}$ selected in $C_{[0]}$ generate entirely $C_{[0]}$. The $4^{\text {th }}$ column of $G$ is selected among one of the other cosets. After this choice, the whole coset is generated. The $5^{\text {th }}$ column of $G$ is selected in one of the 6 other cosets. After this choice, $C_{[0]}$ and three other cosets are generated. It remains four no generated cosets to selecte the last column $G_{6}$ of the coding matrix $\boldsymbol{G}$ of a fully balanced code with $L=L_{\text {min }}$.

\section{CODE PERFORMANCE}

In section 4.2. it was shown that many good published codes are balanced. Only two presented codes in Table II are not balanced :

- The Chen's code with 16 states, 3 transmit antennas obtained by using the rank and determinant criteria.

- The Chen's code with 64 states, 4 transmit antennas and obtained by using the trace criterion.

A systematic computer search in the class of fully balanced codes of type II and III with 16 states and 3 transmit antennas was carried out to detect better fully balanced codes. Table IV shows some parameters of the Chen's code and of a new fully balanced code .

TABLE IV. CODES BASED ON THE RANK AND DETERMINANT CRITERIA

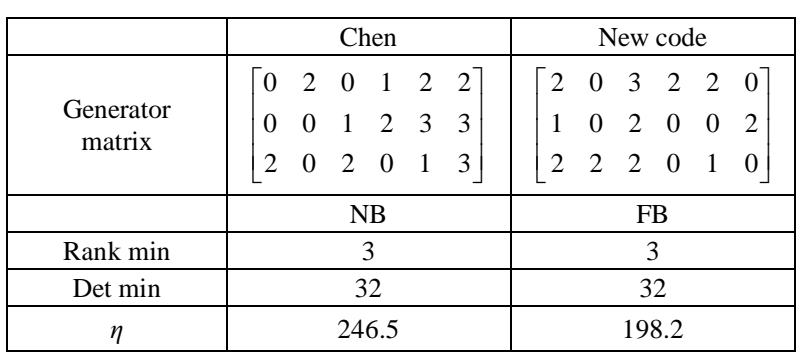

The minimum rank, the minimum determinant and $\eta$ have been evaluated for a 8-bit input binary sequence. Both fullrank generator matrices have the same minimum determinant but the new code has a lower $\eta$. According to [12], in the case of one receiving antenna, the new code must be better than Chen's code because $\min \{\operatorname{rank}(A)\} . n_{R} \leq 3$.

In the same way, a systematic computer search in the class of balanced codes with 64 states has been effectuated to find code with greater trace than Chen's codes for 3 and 4 transmit antennas. Table $\mathrm{V}$ present two new codes. The trace values of these new balanced codes are respectively 32 and 40 versus 28 and 38 for Chen's code.

The performance of the new STTCs and the best Chen's code was evaluated by simulation. For each code, a frame of 130 QPSK symbols was used. We assume that the channel fading coefficients are independent samples of a complex Gaussian process with zero mean and variance 0.5 per dimension. The simulation has been effectuated over slow fading channels. Fig. 2 shows the performance of the QPSK 16-state codes with 3 transmit antennas and only 1 received antenna. In this case, rank and determinant criteria must be 
predominant. This new fully balanced code outperform to the corresponding best known code by Chen.

TABLE V. CODES BASED ON THE TRACE CRITERION

\begin{tabular}{|c|c|c|c|c|c|c|c|c|}
\hline$n_{T}$ & States & Code & \multicolumn{4}{|c|}{$G$} & & $d_{\min }^{2}$ \\
\hline 3 & 64 & $\begin{array}{l}\text { New } \\
\text { code }\end{array}$ & $\begin{array}{l}2 \\
0\end{array}$ & & & $\left.\begin{array}{l}3 \\
2 \\
1\end{array}\right]$ & FB & 32 \\
\hline 4 & 64 & $\begin{array}{l}\text { New } \\
\text { code }\end{array}$ & & & & $\left.\begin{array}{l}0 \\
2 \\
2 \\
2\end{array}\right]$ & B & 40 \\
\hline
\end{tabular}

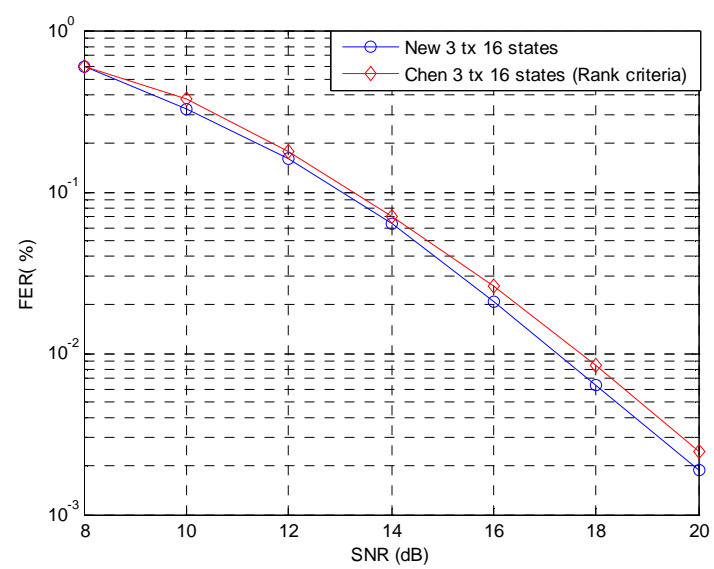

Figure 2. Performance of QPSK STTC with 3 Tx / 1 Rx antennas

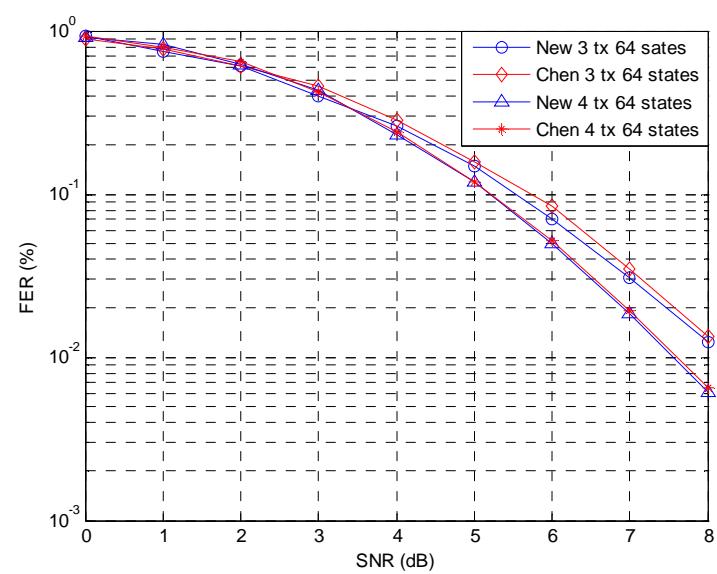

Figure 3. Performance of QPSK 64 states STTC with 3 Tx \& 4 Tx / 2 Rx antennas
In fig 3. the performance of new codes with 64 states for 3 and 4 transmit antennas is compared to corresponding Chen's code given in Table II . The simulation is effectuated with 2 receive antennas. For 3 and 4 transmit antennas, the new balanced code outperform the code prposed by Chen in [8].

\section{CONCLUSION}

In this paper, a new and simpler method to design QPSK BSTTC for several antennas has been proposed. These codes generate the points of the MIMO constellation with the same probability. It has been shown that most of the good known codes belong to this class of codes. For good no balanced codes, balanced codes with better performance can be found. This method can be simply generalized for any $2^{\mathrm{n}}$-PSK modulation, any number of transmit antennas and any number of states of the encoder.

\section{REFERANCES}

[1] V. Tarokh, N. Seshadri, and A.R. Calderbank, "Space-time odes for high data rate wireless communication: Performance criterion and code construction," IEEE Trans. Inform. Theory, vol. 44, no. 2, pp. 744-765, March 1998

[2] Z. Chen, J. Yuan, and B. Vucetic, "Improved space-time trellis coded modulation scheme on slow fading channels," Electron. Lett., vol. 37, no. 7, pp. 440-441, March 2001.

[3] W. Firmanto, B.S. Vucetic, and J. Yuan, "Space-time TCM with improved performance on fast fading channels," IEEE Commun. Lett., vol. 5, no. 4, pp. 154-156, April 2001.

[4] S. Baro, G. Bauch, and A. Hansmann, "Improved codes for space-time trellis-coded modulation," IEEE Commun. Lett., vol. 4, no. 1, pp. 2022, Jan. 2000.

[5] Yi Hong and A. Guillen i Fàbregas, "New Space-Time Trellis Codes for Slow Fading Channels," in Proc. IEEE VTC 2006-Spring, vol. 3, pp. 1492 -1496, May 2006.

[6] Y.S. Jung and J.H. Lee, "Improved space-time trellis codes using expanded signal set in slow Rayleigh fading channels," in Proc. IEEE ISIT'2002, p. 248, June 2002.

[7] M. Crussiere, J.M. Auffray, and J.F. Helard, "Comparison of STTCM over slow and fast Rayleigh fading channels," in $4^{\text {th }}$ IEEE Workshop on SPAWC 2003, pp. 249-253, June 2003.

[8] Z. Chen, B.S. Vucetic, J. Yuan, and Ka Leong Lo, "Space-time trellis codes for 4-PSK with three and four transmit antennas in quasi-static flat fading channels," IEEE Commun. Lett., vol. 6, no. 2, pp. 67-69, Feb. 2002.

[9] Thi Minh Hien Ngo, G. Zaharia, S. Bougeard and J.F. Helard, "4-PSK Balanced STTC with Two Transmit Antennas", Vehicular Technology Conference, pp. 1693-1697, 2007 IEEE 65 ${ }^{\text {th }}$ VTC2007-Spring.

[10] Thi Minh Hien Ngo, G. Zaharia, S. Bougeard and J.F. Helard, "A new class of balanced 4-PSK STTC for two and three transmit antennas ", in IEEE 8th Workshop on Signal Processing Advances in Wireless Communications, SPAWC 2007, , pp. 1-5, June 2007.

[11] Thi Minh Hien Ngo, G. Zaharia, S. Bougeard and J.F. Helard, "Design of Balanced QPSK Space-Time Trellis Codes for Several Transmit Antennas" Proc. IEEE ISSCS 2007, vol. 2, pp. 1-4, 13-14 July 2007.

[12] Chen Liao, V.K. Prabhu "Improved code design criteria for space-time trellis codes overs quasi-static flat fading channels", $6^{\text {th }}$ Workshop on SPAWC 2005, pp. 7-11, 5-8 June 2005.

[13] D. Bernier, F. Chan "Improved space-time trellis codes with three and four transmit antennas" Canadian conference on Electrical and Computer Engineering 2004, vol. 4, pp. 2089-2093, May 2004. 